\title{
Developing Cultural Competencies through Transnational Learning Experiences in Active Online Learning Environments
}

\author{
http://dx.doi.org/10.3991/ijep.v4i5.3534 \\ D. May ${ }^{1}$, K. Wold ${ }^{2}$ and S.L. Moore ${ }^{2}$ \\ 1 TU Dortmund University, Dortmund, Germany \\ ${ }^{2}$ University of Virginia, Charlottesville, VA, USA
}

\begin{abstract}
In today's world cultural competency becomes more and more important in order to act successfully in the modern economy. Nevertheless it is often difficult to engage students in immersive international experiences that develop inter-cultural awareness. Especially undergraduate engineering students have to deal with packed curricula with little or no room for languages and an often unforgiving structure that puts them a year out of course sequences if they do travel for a study abroad. In this paper, we examine how an online engineering education can be a transformational factor in this challenge. We present a joint online engineering course designed and implemented by the University of Virginia (UVa) and the TU Dortmund University (TU) with students from both countries. In addition to the course concept itself we explain our findings on student interaction throughout the course.
\end{abstract}

Index Terms-intercultural competency; Engineering Education; transnational course setting; online engineering course

\section{INTRODUCTION}

The landscape of the engineering workplace has changed over time, and today graduates increasingly face a workplace in which they work on projects with global implications or positions in multinational corporations in which they must meet, plan, and work with peers around the world, often not in person and with the resources available to each at their own location [1]. The systems these students will work on are complex with implications for multiple countries. That requires being able to think and plan beyond a local focus. For example, when a nuclear plant in one country experiences problems, the whole world shares in the consequences as policies and planning around the world respond to events outside their own borders. We all could see this kind of an impact during the tsunami on the Fukushima-Daiichi plant in Japan. According to technology ethicist Barbour [1], as we scale up our technological systems, so too must we scale up our frameworks for ethics in decision making and planning to consider not just the technical implications but also the cultural, environmental, and global. This ability to consider larger, more complex systems and their longterm consequences is a critical skill for professional engineers and has been articulated as a priority outcome for engineering programs in the United States, formally adopted as a major programmatic outcome by the Accreditation Board for Engineering and Technology for US programs [2]. But in order to be aware of and prepared to address this international aspect of engineering, students have to develop global competence. That does not happen unless students acquire experience in collaborating with international peers, and in a fashion consistent with how they will do so on the job.

In Fall 2011 (and again in Fall 2012), the University of Virginia (UVa) and TU Dortmund University (TU) designed and delivered a course that focused on the professional role of engineers in the design of global technological systems. In working together on an international offering of such a course, the instructors from UVa and TU specifically sought to emphasize cultural themes throughout, looking at a broad range of examples that played out differently in different cultures and reading cross-cultural comparisons. However, identifying the content proved to be the simple part in designing an online, international course for students from multiple institutions and countries. Both instructors recognized the need for careful design in order to navigate through the logistical, structural, cultural, and assessment challenges of such a course [3],[4],[5] - that design, the results of the Fall 2011 and the repetition of Fall 2012, along with next steps, are detailed herein. (See also [6],[7])

\section{PRELIMENARY ORGANIZATIONAL AND}

\section{DiDACTICAL CONSIDERATIONS FOR THE COURSE DESIGN}

In higher education there are different possible models for a course design process. With this paper we will show how we combined two different approaches - the Instructional Design and the Constructive Alignment - for the development of an online engineering course, beginning with the general conditions for the course and ending with the explicit course design in detail. 
PAPER

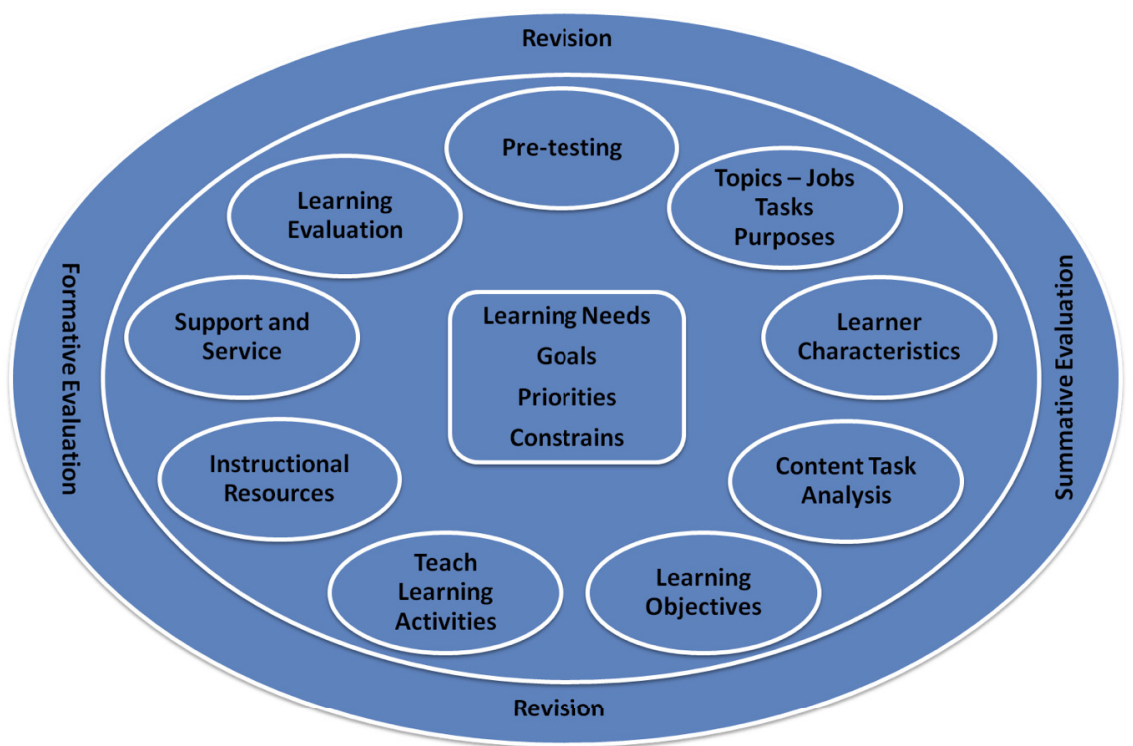

Figure 1: The Instructional Design model from Morrison, Ross, and Kemp [7]

The system of Instructional Design is a complex model for how to systematically design instruction and improve it over time, drawing from theoretical bases such as general systems theory. Over the years, a suite of models have been developed based on differing paradigms of thought such as behavioral, cognitive, and constructivist models. In general, the parts of any model of instructional design remain largely the same (for a review of the models as well as the historical process of development of instructional systems design, see [8]. For our purposes, we have used the model from Morrison, Ross, and Kemp [9] in depth to explore how that aided in the design of an international online learning experience, although it should be noted that there are other equally viable options (see Fig.1).

A systematic instructional design process maps out the different decision points and considerations that should go into the development of any effective learning environment. For transnational education, these models can be particularly helpful as they highlight the need to define learners' characteristics, expectations, and past experiences as well as define institutional expectations. These models additionally spark discussion regarding constraints such as scheduling differences, time zones, technological infrastructures, and of course, the model

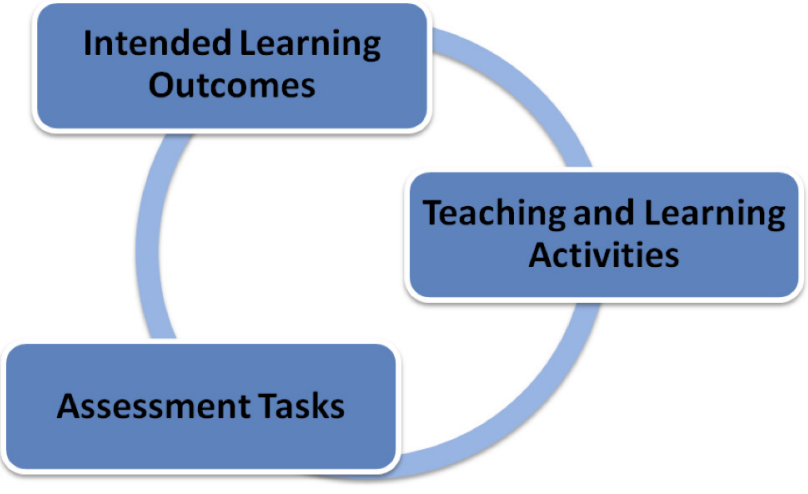

Figure 2: The three elements in an aligned course [8] highlights the learning needs and goals. By working through certain details first, the shape of the course can be better informed as refined objectives are articulated and appropriated activities or strategies can emerge from understanding the learners, the constraints, and so forth.

With the Constructive Alignment we took into account another fundamental concept in higher education [10]. The central message within this concept is the need for an alignment of the three central course design scopes - the intended learning outcome, the teaching and learning activities, and the examination (see Fig. 2). Following this model, a course design process circulates between these three components and takes them into account - beginning with the intended learning outcome. This consequently means, that the course's concept has to be designed in a way that students are able to develop the central competences - defined as intended learning outcomes (ILOs) - the course wants them to develop. A good help in order to couch the ILOs in terms is the usage of explicit verbs describing the outcomes from the students' perspective and indicating the level of understanding and the performance the students are expected to achieve during the course. The latter in particular is a crucial point in describing learning outcomes, because the simple word "understand" is often used in the descriptions of higher education courses' aims but just "understand" does not clearly outlines to the students what they are expected to do. A better learning outcome's description would use the words "explain", "explain in depth", "reflect on", or "evaluate".

In order to accomplish the ILOs, the teaching and learning activities (TLAs) must consequently provide the opportunity for the students to develop their personal competence level and mind-set towards the outcomes. In addition to that the course's assessment tasks (ATs) also have to address these ILOs and need to allow the students to show their developed competences. This includes the opportunity to perform in the examination process as they did during TLAs and as the ILOs expected them to do. The verbs used for describing the ILOs can help for the whole course design process because they are good indicator for choosing the right TLAs and ATs. The 


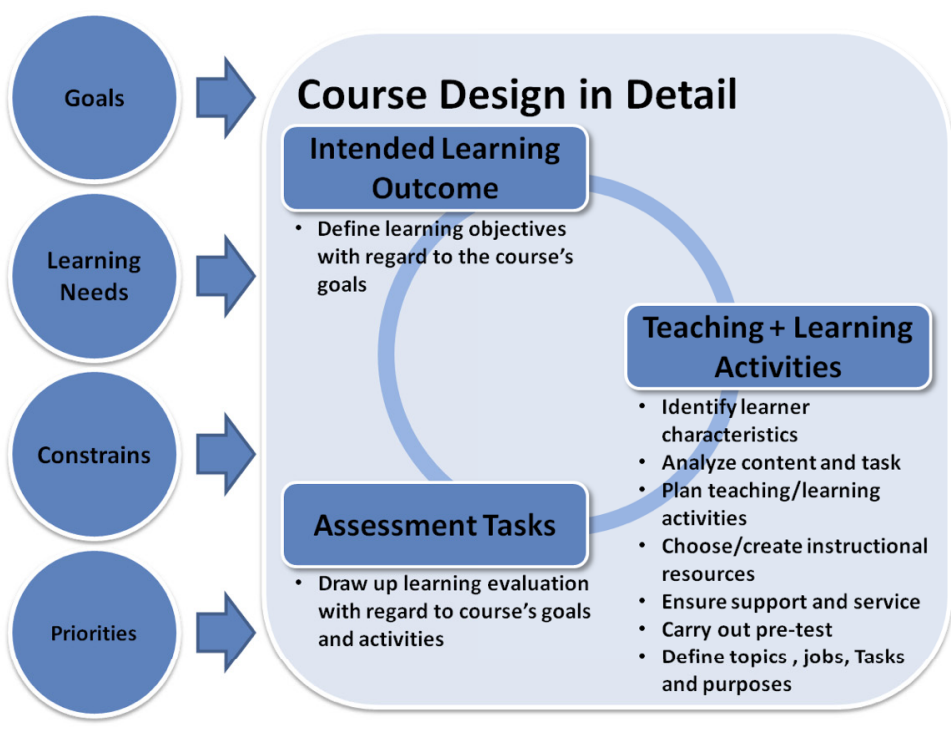

Figure 3: Instructional Desing in combination with Constructive Alignment

following short example explains a course, which is designed with all three elements aligned: If the intended learning outcome for an engineering course is, that "the students should be able to communicate effectively in a transnational team solving a given problem" the TLAs have to address exactly this performance. Consequently the students should practice to work in transnational teams throughout the whole course and the examination should give the opportunity to do so, too. Only a simple multiple choice at the end of the course asking for different aspects of transnational communication wouldn't make sense. We will come back to this example later in context with the actual course description.

After explaining the two didactical and organizational concepts we used for our course design we go on with the description of how we combined them (see Fig.3). As the instructional design model begins with the different parameters for the course we started with them and defined in a first planning phase the goals, learning needs, priorities, and constrains. After that we took the step in the actual course design in detail and took the constructive alignment into account for the second phase. Beginning with the actual intended learning outcomes we started to design the different teaching and learning activities and ended up with the course examination. At this point we came back to the instructional design model and tried to focus on the aspects displayed around the center in Fig.1. The process was not as linear as the figure may indicate, but it was more a back and forth process between the different phases [8], [9]. In the following we will focus on the three components given by the constructive alignment and explain the respective course design aspects.

\section{THE COURSE DESIGN}

\section{A. Intended Learning Outcomes}

Following our design process model we defined the intended learning outcomes after looking at the parameters but before planning the actual teaching and learning activities. As mentioned above the overall goal for the course was to develop intercultural competency through an intercultural learning experience with US students and German students in context with the future of engineers' work. We could divide this goal in more detailed objectives by defining the explicit intended learning outcomes for the students. These are the goals:

- Explain the complex system of technology with respect to technological, organizational, and cultural aspects using explicit examples.

- Reflect on the global perspective of an engineer's work in a globalized world

- Work effectively together in transnational teams, which includes discussing critically about given topics and working out presentations on it.

- Communicate in transnational teams, stating the own opinion and respecting the others'.

- Develop both the interpersonal and technical skills necessary for international collaboration on teams.

- Develop a framework for thinking about and working on complex, global challenges.

These ILOs helped us in a first step to make clear what we expected from the prospective course design. In a second step it was an adequate working mode in order come from the general course idea and defining what was to be done in the actual design of the teaching and learning activities. These activities we will explain in the next part. As to be seen in Fig. 3 the process' step in context with the teaching and learning activities not only includes the activities' design. Defining the learner characteristics, the content selection and of course the instructional resources are part of it. Because of that the following part begins with the learner description.

\section{B. Teaching and Learning Activities}

The typical class session included a mix of presentation by the lead instructors along with either polling the students on understanding of the readings and discussions. However, not all class sessions were lecture and discussion. To move beyond content delivery into content engagement, we developed class activities, that were sequenced to move the students from learning about each other, over communicating with each other, to participating in an authentic, immersive case study so that - by the end of the course - they had to actually work on a problem together (see Fig. 4). 


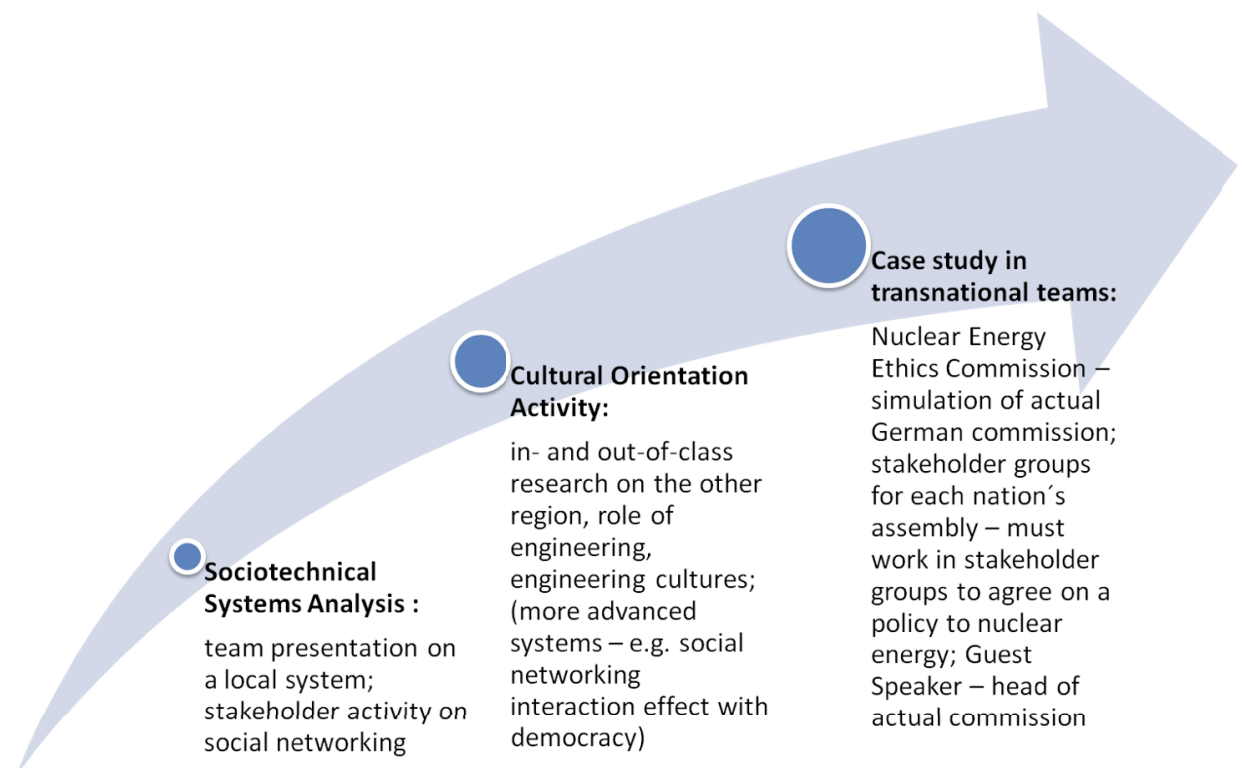

Figure 4: Series of activities during the course to guide the students to work in transnational teams

Sociotechnical Systems Analysis: The first set of activities are explicitly geared towards scaffolding the students to identify cultural components of systems, collaborate with a partner or team, and understand the perspectives as well as the influence of different "relevant social groups" [11]. First, students had to work in teams of two or three in order to analyze socio-technical systems with local implications for their communities. That included clearly identifying the cultural aspects of the system and how those shaped or were shaped by the technical aspects (the notion of "mutual shaping" from sociotechnical systems analysis). By doing so the students demonstrate early on how well they understand and can identify those cultural aspects. The students also engaged in a series of group discussion activities. These discussion activities occurred at least once a week; some were whole class discussions, others were designed to break students into meaningful groups to discuss the topic from different perspectives. For example, we had an activity on the role of social networking technologies in Middle East protests from 2011. Students read or watched news reports from multiple sources (US and abroad) and were then put into discussion groups representing different stakeholder in the debate. A feature in the collaboration software made it easy for us to mix students in the groups every time: break-out rooms were created, and students were placed in these breakout rooms randomly (the software randomly sorts the students into rooms), so every time they could be working with a totally different group of individuals with any possible combination between US and Germany. For course's success it was crucial to get students used to work together and warmed up for talking to each other for effective engagement in the final activity, even if they felt nervous about making mistakes.

Cultural Orientation Activity: The second major activity was a cultural orientation activity in which they researched, read articles, and learned about the other country, state, and university. For each group, the instructor from the other country facilitated that particular course session. Students had readings and web tours to do outside of class, and in class they heard first-hand experiences and guest lectures on the role of engineering in the both countries. By the end, they were expected to understand more about the culture, the history, and the role of engineering in the other location. Thus, we learned about "culture" in a broad sense but also discussed engineering cultures as well [12].

Case study in transnational teams: As a third activity our pinnacle activity with regards to the course's goals -, we created a case study to work on. In this case study we wanted them to come together in transnational teams, so that they found it necessary to communicate and act across their cultural borders and to gain the competence in working effectively in an international team. For such a case study, it was important to choose a topic that is of prime importance for the students of both countries and shows the global aspect of engineering. In addition to that we wanted to work on a case study, which reflects an actual discussion on engineering and shows that engineering work has to be seen in a complex interconnection with different stakeholders like politics or society. By looking at these needs it became obvious that future energy supply could be such a topic of significant joint and international interest in engineering. In seeking to identify a precise topic, we selected the current discussions in Germany and the US on nuclear energy. Working on that topic gave a broad range of opportunities to connect it with the overall course subjects and goals.

We decided to design our pinnacle activity for the class in a similar way Germany handled the political discussion on future energy supply in 2011. So we designed the "Nuclear Energy Ethics Commission" exercise. By doing this it was possible to connect a technical engineering topic - which energy supply without a doubt is - with a lot of side issues that have an impact on the discussion as well. Depending on which stakeholder groups take part in such an ethics commission, you can address questions concerning technical, future, social, religious, or even financial aspects. Even the word "ethics" in the case study's name underscores that the students had to think beyond just the technical aspects.

For the Nuclear Energy Ethics Commission, students were split into two assemblies: a US assembly and a German assembly (see Fig. 5). Both assemblies had students from both countries that were carefully assigned 

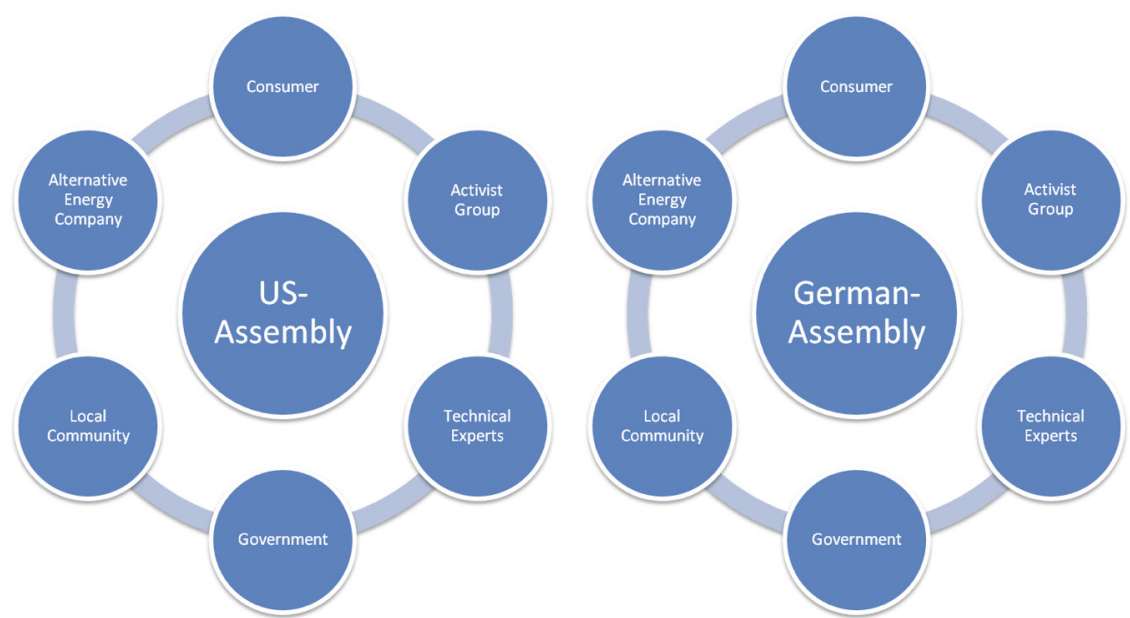

Figure 5: Two assemblies during the Nuclear Energy Ethics Commission exercise

to ensure equal distribution. Within each assembly, students were put into stakeholder groups (5-6 stakeholder groups per assembly). The students had to work with the members of their own stakeholder groups to clearly identify their stakeholders' interests and work out a stakeholder statement they presented in a first step to the rest of their assembly. After the stakeholder groups presented their position, each assembly had to develop a joint recommendation to the respective government on how to go on in questions of future energy supply.

This activity occurred over four class periods. Students were allowed to work together outside of class using the same collaboration software in self-organized group work. After the activity, we had a debriefing period in which we discussed the exercise.

For the instructional resources, we needed an online technology platform, which allowed the students to all be present from wherever they are and have equal access to communication and collaboration tools. As explained above, for most of the course sessions the students were expected to discuss and work together on examples. So they had to be split into discussion groups and provided with discussion questions intended to get them thinking through the course content. We wanted those discussion groups to include students from both countries. And clearly, for the Nuclear Energy Ethics Commission, the students had to work together in cross-country teams, both

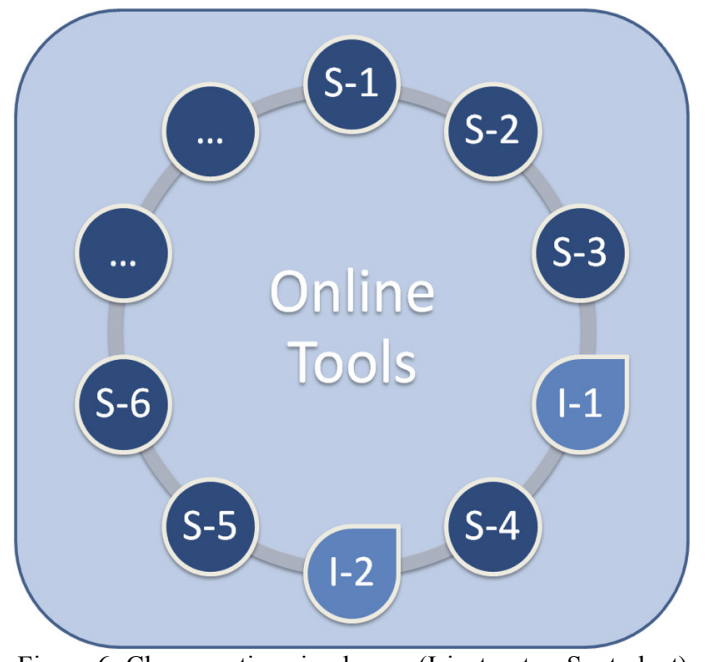

Figure 6: Class meetings in plenum (I-instructor, $\mathrm{S}=$ student) in and out of class. All these aspects were requirements for the instructional platform.

As instructional resource for this course we chose an online environment that allowed students to connect from wherever they are, called Blackboard Collaborate. The Collaborate environment is fitting for this course because students sign in from a computer wherever they are and have tools that allow them audio, video, desktop sharing, and whiteboard capabilities among other features. For the class meetings, the instructor can moderate the environment, students can raise their hands, and they can post comments or questions in a text area (see Fig. 6). It also includes polling and quizzing features as well as the ability to send students to breakout rooms (see Fig. 7). Within those breakout rooms, students have all the same tools available and the instructors can go from room to room to check in on groups and talk with them. Class sessions can also be recorded so students can go back and revisit any day's topics. Students can also use this environment to plan, discuss, share, and then give individual or joint presentations. They can create their own meetings outside of class time to meet as a group and self-organize their group projects, all using the same tools as the course so there is no additional learning curve just to be able to collaborate.

In addition to the live class environment, all such as the course content, syllabus, and readings were loaded

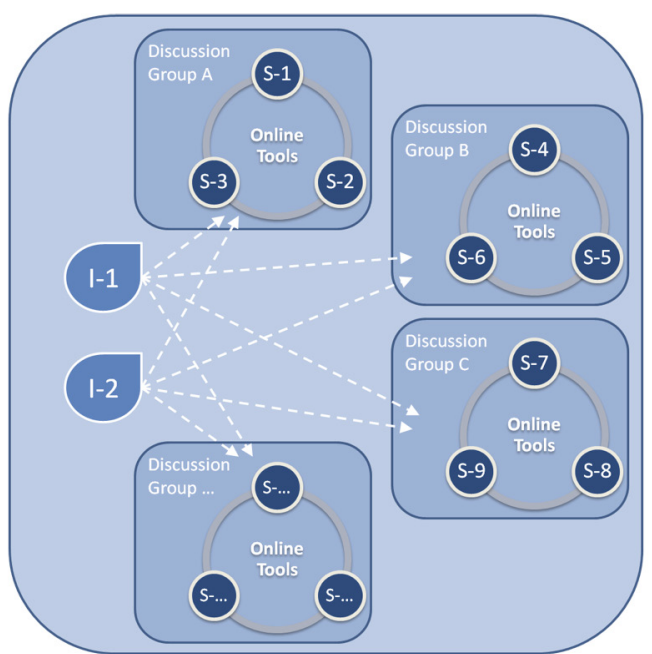

Figure 7: Group discussions in breakout rooms (I=Instructor, $\mathrm{S}=$ Student) 
PAPER

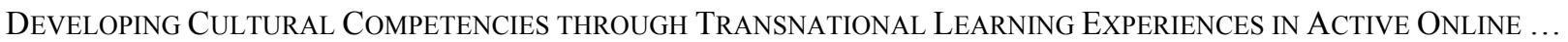

through a course shell using UVa's learning management system (LMS), Collab (distinct from the synchronous meeting tool named Collaborate). UVa's Collab is an instance of the Sakai open-source environment. It includes a Resources area that houses course readings and documents as well as Assessments and Quizzes, Discussion Board, Wiki, and other common features for an LMS. The Collaborate sessions are also integrated into the course calendar in the course's Collab site, so the Collab site serves as the main portal to all the material and sessions.

\section{Assessment Tasks}

As we discussed under learner characteristics, the students' expectations were quite heterogeneous. This proved to be one of the details requiring a lot of attention. For UVa students, we could not decrease the number of papers, presentations, or exams because these are tied to specific standards and accreditation expectations. However, we were concerned that students from Germany would respond very negatively to the volume of deliverables and tests for evaluation since it is so radically different from what they are used to. We used the scheduling offset to our advantage to address portions of this. The UVa students completed one of their papers and one of their exams before the German students joined the class. We reduced the number of exams for UVa students to two total (a mid-term and a final). Their second paper was due just shortly after the German students join. The third paper was required for all of the students. As for presentations, UVa students gave two presentations prior to the German students joining. The third and fourth presentations were joint presentations with students from both countries in the groups and occurred as part of the Nuclear Energy exercise. As a lead in to this, we had several in-class activities on which students from both countries had to discuss, put together a brief presentation, and jointly present live in the synchronous environment. All of the students participated in a final reflection activity conducted during class time. The two instructors handled grading independently, as the overall percentages and weighting for individual items varied for the UVa students vice the German students.

So the evaluation of the TU students for example mainly was composed of grades for general performance and participation in the course $(40 \%)$, the sociotechnical system presentation $(15 \%)$, the ethics commission exercise performance and presentation $(20 \%)$ and the final paper $(20 \%)$. The final $5 \%$ of the course grade the students could achieve with a final multiple choice test. Even if we had to write this test because of curriculum constrains, it was obvious for us that such a written assessment did not fit to the course concept and so we did it but it was not the main important part for the final grading. We showed that grading system at the very beginning to the students so that it was transparent to them that class participation and communication is one of the most important aspects in order to pass the course with a good grade.

\section{Alginment}

Before we change over the students' interaction and feedback we would like to come back to the didactic background explained above. We showed that the constructive alignment was one of the basics we used during the planning process. So it was important for us to have a close look at the interconnections between the intended learning outcome, the learning activities and the assessment. Fig. 8 shows how the alignment worked out in our course. Reading the figure from the left to the right you can see which of the ILOs led to which of the course's activities and how we measured the students' performance. It shouldn't be surprising that the ethics commission exercise corresponds with every ILO. As explained above this activity was our pinnacle activity and the students worked on this exercise for several weeks. During this activity all of the topics we discussed before came together as they had to work in international teams and discussed a technology with global implications from different perspectives. The showed assessment tasks only count for the German students as these tasks differed significantly from group to group and the American students for example had to write more papers.

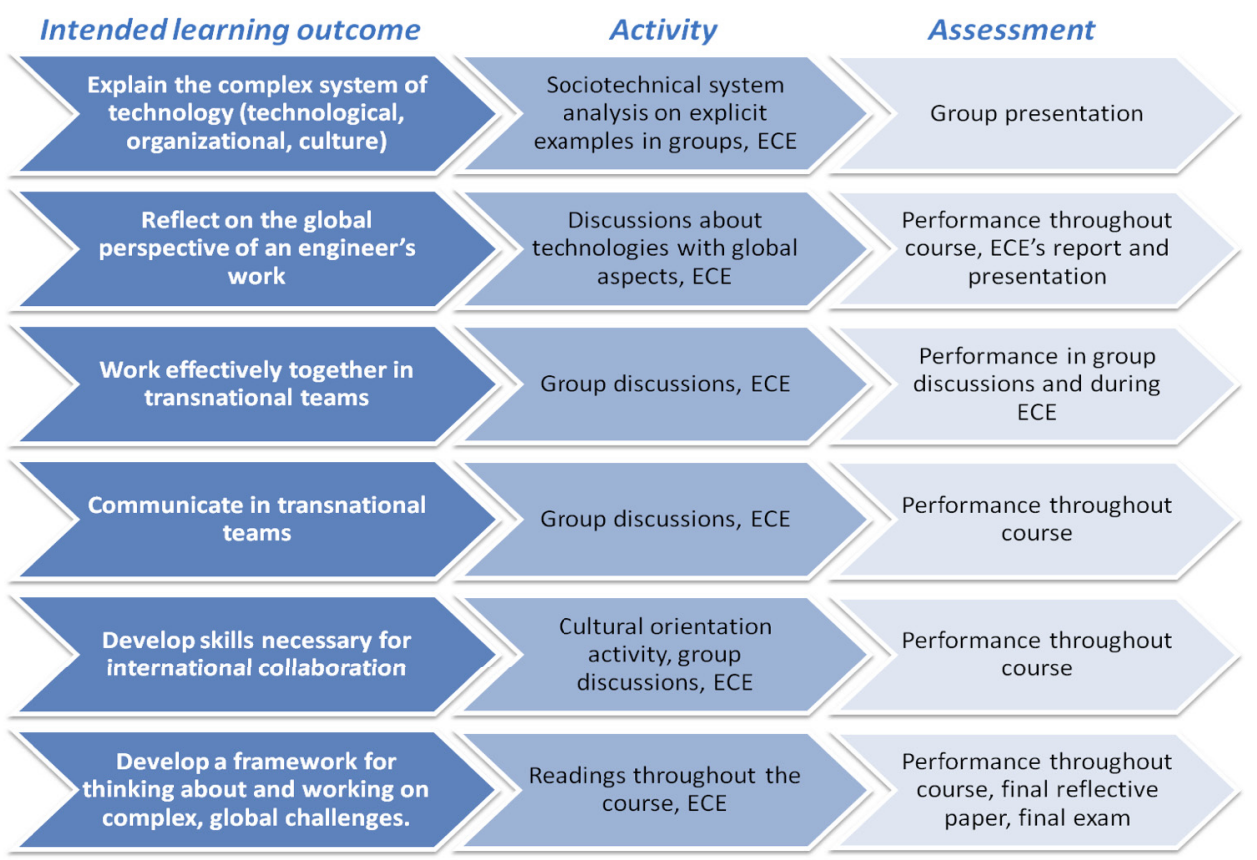

Figure 8: Course Alignment with special regard to the German student group 


\section{STUDENTS' INTERACTION}

The discussion format allowed us to study whether students from both cultures were able to improve their abilities to show global competency. We were looking for whether students were able to visibly better able to show respect to other students, recognize differences, adjust their behaviors, and integrate others' ideas when making group decisions. To do so, interactions and interviews were evaluated using a rubric that illustrated students were at the denial, minimization, acceptance, adaptation, or incorporation stage in their abilities to display global competency. Classifications were then organized chronologically in order to determine whether and how students changed stages throughout the course as well as how they displayed global competency throughout the course.

Through our analysis, we found that most students increased their abilities to display global competency. For instance, most students showed respect to other students by sharing information about their country and increasing their contributions to the group as the simulations continued. The majority of students also increased the ability to recognize differences and not assume that everyone in Germany had one view; German students were less likely to assume U.S. students had one view of the use of nuclear energy.

The majority of students additionally adjusted their behaviors throughout the simulation to improve their interactions with those from other cultures. Ways they did this included providing websites to country information about nuclear energy as well as rephrasing their wording to be understood. Most students increased their abilities to integrate ideas by specifically asking students who seemed hesitant to contribute what they wanted to do and expressing the importance of getting contextual information from others to solve a problem much more than they had at the beginning of the simulations.

A vast minority of students, however, did not believe there were significant differences between the two cultures or did not believe they had significant participation with those from other cultures. While these students all believed the exercise was useful to learn class content, they did not believe it was a significant cultural

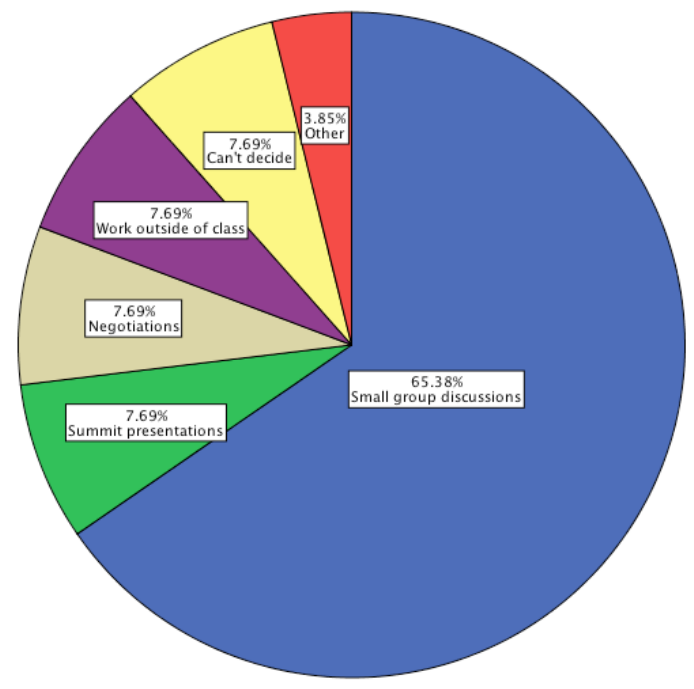

Figure 9: Activities all students believe most facilitated interactiosn with students from other cultures experience. These students were placed in groups where students from other cultures either dropped the course or did not actively participate, which led to frustration among teammates.

Although changes in students' perceived abilities to display global competency were not statistically significant, students' actions throughout the simulations illustrated they did change their behaviors and thought processes in different ways while staying static in others. They also significantly shifted their opinions on roleplaying simulations where the majority of students reported they enjoyed role-playing simulations more than they did before the exercise. Their behavior and comments showed students were more interested in learning from others than learning how to display global competency; however, through working with others, they often consciously or subconsciously learned how to enhance their global competency skills in the process.

Results from the study additionally showed students enjoyed the simulation with over $65 \%$ of students reporting they enjoyed small-group discussions the most (see Fig. 9).

Over $66 \%$ of the students interviewed believed the small group discussions were useful because they liked learning how to adjust how they communicated when working with students from different cultures. All German and U.S. students interviewed agreed it grew easier to communicate as the simulations continued; all also reported having small three- or four-person groups was best in order to become familiar with everyone and have everyone contribute.

Other elements of the simulations were less popular, such as the group presentations. Most students believed the presentations were useful for giving them a place to debate with other cultures. However, some German and U.S. students expressed they believed presentations placed a lot of pressure on people, particularly the German students who were less comfortable spontaneously communicating in English in large-group settings.

One popular component of the simulations was to have one simulation focus on Dortmund and the other focus on Virginia. Many German students reported this was because they felt like they had a lot to contribute for the Dortmund simulation, and all enjoyed working with a topic that was timely and globally controversial.

All but one student expressed interest in participating in a transnational role-playing simulation again. Both German and U.S. students reported it helped them learn the different opinions and ways of thinking of those from different cultures, and all reported it was an interesting experience overall.

\section{CONCLUSION}

With this paper we present an online course design, which provides the opportunity to give students from transnational universities the chance to learn together in one group. Especially for engineering students this will be a key competence because their future work will be more and more global connected and engineers will be forced to work together in transnational teams. With the presented course they are given the opportunity to develop this competence very early in their career.

We showed how course design at the university could work by combining the instructional design model and the 
concept of constructive alignment. With regards to four key parameters - the goals, learning needs, priorities, and constrains - we designed the course more in detail by following the three steps of defining the intended learning outcomes first, the teaching learning activities in a second step and finally the assessment.

Given that the course feedback from the students was almost exclusively positive, we have demonstrated that such a course concept could be an adequate concept in order to bring international students together. Of course, going abroad still is a deeper experience but taking part in such an international course could be at least one step into the direction of thinking globally and such a course provides access to international collaboration to a broader number of engineering students. Future research directions for this course are seen in two areas: (1) developing and measuring a suite of different approaches and activities to develop cultural competency, and (2) on-going work on how we measure and evaluate cultural competency. The activities and design solutions presented in this course do not reflect the only possible design solution for how to leverage distance environments for transnational learning. The obvious variety and differences in approaches from countries around the world suggest that a range of instructional activities may prove effective in different contexts. In addition, this area could likely benefit from other types of instructional technologies, such as simulations or virtual worlds, in which students can either see consequences for their decisions or manage a system to see how it evolves based on their collective decisions. To assess cultural competency as a learning outcome will be the most difficult as well as the most interesting part of our research on this course. As the world and its labor markets more and more merge to only one market, the importance of cultural competency as a learning outcome will steadily increase in the future. The presented course shows one opportunity for universities to face this demand and how to work together on innovative as well as transnational teaching and learning approaches for engineering education. Cultural competency is not a question to be worked on in mono-cultural teams at national universities, but we have to work on it in a crosscultural manner; above all at universities and especially in engineering education. [13]

\section{REFERENCES}

[1] I. Barbour, "Ethics in the age of technology", New York, NY: HarperOne, 1992

[2] J.C. Swearengen, S. Barnes, S. Coe, C. Reinhardt and K. Subramanian, "Globalization and the underraduate manufacturing engineering curriculum", Journal of Engineering Education, 91(2), pp. 255-261, $2002 \quad$ http://dx.doi.org/10.1002/j.21689830.2002.tb00700.x

[3] G. Augusti, "Accreditation of engineering programmes: European perspectives and challenges in a global context", European Journal of Engineering Education, 32(3), pp. 273-283, 2007 http://dx.doi.org/10.1080/03043790701276742
[4] J. Borrego, "Roadmap for a successful transition to an online environment", Contemporary Issues in Education Research, 3(5), pp. 59-66, 2010

[5] K. M. Passino, "Educating the humanitarian engineer", Science \& Engineering Ethics, 15(4), pp. 577-600, 2009 http://dx.doi.org/10.1007/s11948-009-9184-8

[6] Moore, St., D. May; "Global Perspectives for Engineering Students: The Use of Active Online Environments for CrossCultural Learning"; conference proceedings of 'ED-MEDIA 2012 - World Conference on Educational Multimedia, Hypermedia \& Telecommunications' der Association for the Advancement of Computing in Education (AACE); Denver (Co, USA); 25. 29.06.2012; S. 1773 - 1783; ISSN/ISBN: 1-880094-95-9

[7] Moore, St., D. May; "Transnational Collaborative Learning for Engineering Students through Active Online Environments Developing "global perspective" for US and German students"; in proceedings of "ICL 15th International Conference on Interactive Collaborative Learning and 41st International Conference on Engineering Pedagogy'; Villach (Austria); 26.-28.09.2012; ISBN:978-1-4673-2426-7

[8] K.L. Gustafson \& R.M. Branch, "Survey of instructional development models (4th ed.)", ERIC Clearinghouse on Information \& Technology, Syracuse University, NY: Syracuse, 2002

[9] G. Morrison, S. Ross \& J. Kemp, "Designing effective instruction", $5^{\text {th }}$ ed. New York: Wiley, 2006

[10] J.B. Biggs \& C. Tang, "Teaching for quality learning at university. What the student does", 3rd ed., Maidenhead: McGraw-Hill, pp. 50 and following, 2007

[11] T.J. Pinch, and W. E. Bijker, "The social construction of Facts and artifacts: Or how the sociology of science and the sociology of technology might benefit each other" In W. E. Bijker, T. P. Hughes and T. J. Pinch (Eds.), The Social Construction of Technological Systems: New Directions in the Sociology and History of Technology (pp. 17 - 50). Cambridge, Massachusetts: The MIT Press, 1987

[12] G. Downey \& J. Lucena, "National identities in multinational worlds: Engineers and "engineering cultures", International Journal of Continuing Engineering Education and Lifelong Learning, $\quad 15(3-6), \quad$ pp. 252-260, 2005 http://dx.doi.org/10.1504/IJCEELL.2005.007714

[13] Moore, St., D. May, K. Wold "Developing Cultural Competency in Engineering through Transnational Distance Learning" in $\mathrm{R}$. Hogan (ed.); 'Transnational Distance Learning and Building New Markets for Universities'; IGI Global; Hershey (PA/USA); 2012; S. 210-228 http://dx.doi.org/10.4018/978-1-4666-0206-9.ch013

\section{AUTHORS}

D. May is with Center of Higher Education at TU Dortmund University in Dortmund, Germany (dominik.may@tu-dortmund.de).

K. Wold is with the Curry School of Education at the University of Virginia in Charlottesville, Virginia, USA. (kaw4qy@virginia.edu).

S. L. Moore is with Curry School of Education at the University of Virginia in Charlottesville, Virginia, USA. (slm6un@virginia.edu).

This article is an extended and modified version of a paper presented at the CISPEE 2013 conference, held October 31 - November 01, 2013, in Porto, Portugal. Article received February 02, 2014. Published as resubmitted by the authors 07 March 2014. 